\title{
On the analytic calculation of multiple integrals in electromagnetics
}

\author{
L. Knockaert*
}

\begin{abstract}
Making use of a powerful Gauss divergence theorem and a new Gauss bi-divergence theorem, we transform a high dimensional moment integral into a lower dimensional boundary integral. This allows in most pertinent cases to replace the original singular multiple integral by a reduced nonsingular multiple integral, which can be evaluated by means of standard analytic or numerical quadrature techniques. Some important electromagnetic cases are treated to indicate the strength and versatility of the proposed method.
\end{abstract}

\section{INTRODUCTION}

The numerical evaluation of multiple integrals with singular kernels arises quite naturally in the method of moments solution [1] of electromagnetic scattering problems. Most of the methods currently in use perform preliminary calculations to obtain analytic or quasi-analytic expressions for the integrand [2], most often by invoking a Gauss theorem tailored to the problem at hand, while afterwards employing numerical quadrature techniques to calculate the remaining non-singular integrals.

Here we follow another approach. Starting with the kernel of the singular integrand, and making use of a powerful Gauss divergence theorem [3] and a new Gauss bi-divergence approach, we transform the $2 d$-dimensional moment integral into a $(2 d-2)$-dimensional boundary integral. This allows in most pertinent cases to replace the original singular multiple integral by a reduced non-singular multiple integral, which can be evaluated by means of standard analytic or numerical quadrature techniques [4]. Pertinent electromagnetic applications include moment integrals for the Coulomb and retarded Coulomb interactions.

\section{GAUSS DIVERGENCE THEOREM}

Let us start with the calculation of a multiple integral over $\Omega \subset R^{d}$ with $\Omega$ compact and simply connected, i.e.,

$$
I(F)=\int_{\Omega} F(\mathbf{x}) d V
$$

*Dept. INTEC-IBCN-IBBT, Ghent University, G. Crommenlaan 8, PB 201, B-9050 Gent, Belgium, e-mail: luc.knockaert@intec.ugent.be, tel.: +3292643328, fax: +3292649969 . This work was supported by a grant of the Research Foundation-Flanders (FWO-Vlaanderen)
If we could find a vector field $\mathbf{G}(\mathbf{x})$ such that

$$
\operatorname{div} \mathbf{G}(\mathbf{x})=F(\mathbf{x})
$$

then, by virtue of Gauss' theorem, we can write

$$
I(F)=\int_{\partial \Omega} \mathbf{n}_{\mathbf{x}} \cdot \mathbf{G}(\mathbf{x}) d S
$$

where $\mathbf{n}_{\mathbf{x}}$ is the outward unit normal to the surface $\partial \Omega$. Note that a related Gauss theorem for the gradient is :

$$
I(\nabla F)=\int_{\partial \Omega} F(\mathbf{x}) \mathbf{n}_{\mathbf{x}} d S
$$

Let us first try to calculate the integral $I(F)$ for $F(\mathbf{x})$ a monomial, i.e.,

$$
F(\mathbf{x})=F_{\alpha}(\mathbf{x})=\prod_{k=1}^{d} x_{k}^{\alpha_{k}}
$$

where the powers $\alpha_{k}$ are natural numbers. Now it is easy to prove that

$$
\operatorname{div}\left[\mathbf{x} F_{\alpha}(\mathbf{x})\right]=\left(d+\sum_{k=1}^{d} \alpha_{k}\right) F_{\alpha}(\mathbf{x})
$$

Hence for $F=F_{\alpha}$ we have

$$
I\left(F_{\alpha}\right)=\frac{1}{d+\sum_{k=1}^{d} \alpha_{k}} \int_{\partial \Omega} \mathbf{n}_{\mathbf{x}} \cdot \mathbf{x} F_{\alpha}(\mathbf{x}) d S
$$

Next, it is also easy to prove that

$$
\int_{0}^{1} t^{d-1} F_{\alpha}(t \mathbf{x}) d t=\frac{F_{\alpha}(\mathbf{x})}{d+\sum_{k=1}^{d} \alpha_{k}}
$$

In other words we have proved that

$$
\int_{\Omega} F_{\alpha}(\mathbf{x}) d V=\int_{\partial \Omega} \mathbf{n}_{\mathbf{x}} \cdot \mathbf{G}_{\alpha}(\mathbf{x}) d S
$$

where

$$
\mathbf{G}_{\alpha}(\mathbf{x})=\mathbf{x} \int_{0}^{1} t^{d-1} F_{\alpha}(t \mathbf{x}) d t
$$

Since this is valid for all monomials $F_{\alpha}(\mathbf{x})$, and $\Omega$ is compact, we can therefore generalize formula (3), 
in virtue of the Stone-Weierstrass theorem, to the space of all continuous functions over $\Omega$, i.e.,

$$
I(F)=\int_{\partial \Omega} \mathbf{n}_{\mathbf{x}} \cdot \mathbf{x} d S \int_{0}^{1} t^{d-1} F(t \mathbf{x}) d t
$$

Note that $\mathbf{n}_{\mathbf{x}} \cdot \mathbf{x}$, considered as a weight function over $\partial \Omega$, is also well-known in the study of the Dirichlet eigenproblem for $\Omega$, since the Dirichlet eigenfunctions $\Phi_{k}(\mathbf{x})$ and eigenvalues $\lambda_{k}$ are related by Rellich's identity [5] :

$$
2 \lambda_{k} \int_{\Omega} \Phi_{k}^{2} d V=\int_{\partial \Omega} \mathbf{n}_{\mathbf{x}} \cdot \mathbf{x}\left(\frac{\partial \Phi_{k}}{\partial n}\right)^{2} d S
$$

Also, since the weight function $\mathbf{n}_{\mathbf{x}} \cdot \mathbf{x}$ is often positive, formula (4) might provide useful quadrature formulas for $\Omega$ starting from quadrature formulas for $\partial \Omega$, in the same vein as the ones developed in [4] p. 43.

In [3] formula (4) was generalized to the translationally invariant formula

$$
I(F)=\int_{\partial \Omega} \mathbf{n}_{\mathbf{x}} \cdot(\mathbf{x}-\mathbf{a}) d S \int_{0}^{1} t^{d-1} F(t \mathbf{x}+(1-t) \mathbf{a}) d t
$$

with a any vector (translational change of center). Moreover, in formula (5), $F$ may be singular, with the proviso that

$$
\lim _{t \rightarrow 0+} t^{d} F(t \mathbf{x}+(1-t) \mathbf{a})=0
$$

It is then easy to apply these formulas to functions of the form

$$
F_{1}(\mathbf{x})=K(\|\mathbf{x}-\mathbf{a}\|)
$$

and

$$
\mathbf{F}_{2}(\mathbf{x})=(\mathbf{x}-\mathbf{a}) K(\|\mathbf{x}-\mathbf{a}\|)
$$

with $\|\cdot\|$ the Euclidean norm. Note that the function $(A+\mathbf{B} \cdot \mathbf{x}) K(\|\mathbf{x}-\mathbf{a}\|)$ can always be written as a superposition of $F_{1}(\mathbf{x})$ and $\mathbf{F}_{2}(\mathbf{x})$.

We have

$I\left(F_{1}\right)=\int_{\partial \Omega} \mathbf{n}_{\mathbf{x}} \cdot(\mathbf{x}-\mathbf{a}) d S \int_{0}^{1} t^{d-1} K(t\|\mathbf{x}-\mathbf{a}\|) d t$

and, since

$$
\mathbf{F}_{2}(\mathbf{x})=\nabla L(\|\mathbf{x}-\mathbf{a}\|)
$$

where

$$
\begin{gathered}
L(u)=\int^{u} t K(t) d t \\
I\left(\mathbf{F}_{2}\right)=\int_{\partial \Omega} L(\|\mathbf{x}-\mathbf{a}\|) \mathbf{n}_{\mathbf{x}} d S
\end{gathered}
$$

\section{GAUSS BI-DIVERGENCE PROACH}

Application of the method of moments [1] in $R^{d}$, in the presence of kernels which only depend on the Euclidean distance between source point and observation point, always leads to the evaluation of $2 d$-fold multiple integrals. In general we need to evaluate the integral

$$
I=\int_{\Omega} \int_{\mho} t(\mathbf{x}) K\left(\left\|\mathbf{x}-\mathbf{x}^{\prime}\right\|\right) b\left(\mathbf{x}^{\prime}\right) d V d V^{\prime}
$$

where $\Omega, \mho \subset R^{d}$ are the compact, simply connected supports of the test function $t(\mathbf{x})$ and basis function $b\left(\mathbf{x}^{\prime}\right)$, respectively. We further suppose that $t(\mathbf{x})$ and $b\left(\mathbf{x}^{\prime}\right)$ vary linearly within their respective supports with respective gradients $\mathbf{p}$ and q. In other words

$$
\begin{aligned}
t(\mathbf{x}) & =t(0)+\mathbf{p} \cdot \mathbf{x} \\
b\left(\mathbf{x}^{\prime}\right) & =b(0)+\mathbf{q} \cdot \mathbf{x}^{\prime}
\end{aligned}
$$

Consider the bi-divergence operator

$$
\diamond \Psi\left(\mathbf{x}, \mathbf{x}^{\prime}\right) \stackrel{\text { def }}{=} \nabla^{\prime} \cdot \nabla \Psi\left(\mathbf{x}, \mathbf{x}^{\prime}\right)
$$

It is easily proved, see $[3,6]$, that the displaymath

$$
\diamond \hat{K}\left(\left\|\mathbf{x}-\mathbf{x}^{\prime}\right\|\right)=K\left(\left\|\mathbf{x}-\mathbf{x}^{\prime}\right\|\right)
$$

is satisfied by the kernel

$$
\hat{K}(r)=-\int_{0}^{r} u d u \int_{0}^{1} K(u t) t^{d-1} d t
$$

Next consider the composite bi-divergence operator

$$
{ }_{b} \diamond_{t} \Psi\left(\mathbf{x}, \mathbf{x}^{\prime}\right) \stackrel{\text { def }}{=} \nabla^{\prime} \cdot\left[b\left(\mathbf{x}^{\prime}\right) \nabla\left[t(\mathbf{x}) \Psi\left(\mathbf{x}, \mathbf{x}^{\prime}\right)\right]\right]
$$

It is seen that

${ }_{b} \diamond_{t} \Psi=b\left(\mathbf{x}^{\prime}\right) t(\mathbf{x}) \diamond \Psi+t(\mathbf{x}) \mathbf{q} \cdot \nabla \Psi+b\left(\mathbf{x}^{\prime}\right) \mathbf{p} \cdot \nabla^{\prime} \Psi+\mathbf{p} \cdot \mathbf{q} \Psi$ Taking $\Psi\left(\mathbf{x}, \mathbf{x}^{\prime}\right)=\hat{K}\left(\left\|\mathbf{x}-\mathbf{x}^{\prime}\right\|\right)$, and defining

$$
\begin{aligned}
J & =\int_{\Omega} \int_{\mho}{ }_{b} \diamond_{t} \Psi d V d V^{\prime} \\
& =\int_{\partial \Omega} \int_{\partial \mho} \mathbf{n}_{\mathbf{x}} \cdot \mathbf{n}_{\mathbf{x}^{\prime}} t(\mathbf{x}) b\left(\mathbf{x}^{\prime}\right) \hat{K}\left(\left\|\mathbf{x}-\mathbf{x}^{\prime}\right\|\right) d S d S^{\prime}
\end{aligned}
$$

and since $\diamond \Psi=\diamond \hat{K}=K$, we obtain

$$
I=J-\mathbf{p} \cdot \mathbf{I}_{1}-\mathbf{q} \cdot \mathbf{I}_{2}-\mathbf{p} \cdot \mathbf{q} I_{3}
$$

where

$$
\begin{aligned}
\mathbf{I}_{1} & =\int_{\Omega} \int_{\mho} b\left(\mathbf{x}^{\prime}\right) \nabla^{\prime} \hat{K}\left(\left\|\mathbf{x}-\mathbf{x}^{\prime}\right\|\right) d V d V^{\prime} \\
\mathbf{I}_{2} & =\int_{\Omega} \int_{\mho} t(\mathbf{x}) \nabla \hat{K}\left(\left\|\mathbf{x}-\mathbf{x}^{\prime}\right\|\right) d V d V^{\prime} \\
I_{3} & =\int_{\Omega} \int_{\mho} \hat{K}\left(\left\|\mathbf{x}-\mathbf{x}^{\prime}\right\|\right) d V d V^{\prime}
\end{aligned}
$$


It is seen that

$$
I_{3}=\int_{\partial \Omega} \int_{\partial \mho} \mathbf{n}_{\mathbf{x}} \cdot \mathbf{n}_{\mathbf{x}^{\prime}} \bar{K}\left(\left\|\mathbf{x}-\mathbf{x}^{\prime}\right\|\right) d S d S^{\prime}
$$

where

$$
\bar{K}(r)=-\int_{0}^{r} u d u \int_{0}^{1} \hat{K}(u t) t^{d-1} d t
$$

since $\diamond \bar{K}\left(\left\|\mathbf{x}-\mathbf{x}^{\prime}\right\|\right)=\hat{K}\left(\left\|\mathbf{x}-\mathbf{x}^{\prime}\right\|\right)$. The vectors $\mathbf{I}_{1}$ and $\mathbf{I}_{2}$ are more difficult to evaluate. After some algebraic manipulations, utilizing the formulas (1)-(2), (6), (7) and exploiting the fact that $\nabla^{\prime} \hat{K}\left(\left\|\mathbf{x}-\mathbf{x}^{\prime}\right\|\right)=-\nabla \hat{K}\left(\left\|\mathbf{x}-\mathbf{x}^{\prime}\right\|\right)$, we obtain, after some tedious calculations :

$$
\begin{gathered}
\mathbf{I}_{1}=-\int_{\partial \Omega} \int_{\partial \mho}\left(\mathbf{q} \cdot \mathbf{n}_{\mathbf{x}^{\prime}}\right) \mathbf{n}_{\mathbf{x}} \tilde{K}\left(\left\|\mathbf{x}-\mathbf{x}^{\prime}\right\|\right) d S d S^{\prime} \\
-\int_{\partial \Omega} \int_{\partial \mho} b(\mathbf{x})\left(\left(\mathbf{x}^{\prime}-\mathbf{x}\right) \cdot \mathbf{n}_{\mathbf{x}^{\prime}}\right) \mathbf{n}_{\mathbf{x}} \breve{K}\left(\left\|\mathbf{x}-\mathbf{x}^{\prime}\right\|\right) d S d S^{\prime}
\end{gathered}
$$
and similarly

$$
\begin{gathered}
\mathbf{I}_{2}=-\int_{\partial \Omega} \int_{\partial \mho}\left(\mathbf{p} \cdot \mathbf{n}_{\mathbf{x}}\right) \mathbf{n}_{\mathbf{x}^{\prime}} \tilde{K}\left(\left\|\mathbf{x}-\mathbf{x}^{\prime}\right\|\right) d S d S^{\prime} \\
-\int_{\partial \Omega} \int_{\partial \mho} t\left(\mathbf{x}^{\prime}\right)\left(\left(\mathbf{x}-\mathbf{x}^{\prime}\right) \cdot \mathbf{n}_{\mathbf{x}}\right) \mathbf{n}_{\mathbf{x}^{\prime}} \breve{K}\left(\left\|\mathbf{x}-\mathbf{x}^{\prime}\right\|\right) d S d S^{\prime}
\end{gathered}
$$
where

$$
\tilde{K}(r)=\int_{0}^{r} \hat{K}(u) u d u
$$

and

$$
\breve{K}(r)=\int_{0}^{1} \hat{K}(t r) t^{d-1} d t
$$

It is seen that $I$, a $2 d$-fold multiple integral, has been written as a sum of $2(d-1)$-fold boundary integrals, which represents a serious computational gain. Note that in the pulse-basis case, when $\mathbf{p}=$ $\mathbf{q}=0$, one simply has $I=J$.

\section{SPECIAL CASES}

An important special case is when the multiple moment integrals are generated by the Coulomb kernel

$$
K\left(\left\|\mathbf{x}-\mathbf{x}^{\prime}\right\|\right)=\frac{1}{\left\|\mathbf{x}-\mathbf{x}^{\prime}\right\|}
$$

Since $K(r)=1 / r$, the other pertinent kernels $\hat{K}(r), \bar{K}(r), \tilde{K}(r)$ and $\breve{K}(r)$ are found by the analytical calculations of (8), (9), (10) and (11). We obtain

$$
\begin{aligned}
\hat{K}(r) & =-\frac{r}{d-1} \\
\bar{K}(r) & =\frac{r^{3}}{3\left(d^{2}-1\right)} \\
\tilde{K}(r) & =-\frac{r^{3}}{3(d-1)} \\
\breve{K}(r) & =-\frac{r}{d^{2}-1}
\end{aligned}
$$

Note that in the pulse-basis case we only need the kernel $\hat{K}(r)$. For $d=2$ we have $\hat{K}(r)=-r$, as was already indicated and discussed in [7].

Another, more general and difficult case is to deal with retarded potentials, in which case the kernel is

$$
K\left(\left\|\mathbf{x}-\mathbf{x}^{\prime}\right\|\right)=\frac{e^{-j k_{0}\left\|\mathbf{x}-\mathbf{x}^{\prime}\right\|}}{\left\|\mathbf{x}-\mathbf{x}^{\prime}\right\|}
$$

It is therefore important and potentially rewarding to calculate the $\hat{K}(r)$ kernel associated with the retarded Coulomb kernel $K(r)=e^{-j k_{0} r} / r$. Application of formula (8) yields

$$
\hat{K}(r)=\int_{0}^{1}\left(\frac{e^{-j k_{0} r t}-1}{j k_{0} t}\right) t^{d-2} d t
$$

Although the kernel $\hat{K}(r)$ defined by the integral (16) is an entire function with easily found series representation

$$
\hat{K}(r)=-r \sum_{m=0}^{\infty} \frac{\left(-j k_{0} r\right)^{m}}{(m+d-1)(m+1) !}
$$

it is seen that the delay information is completely lost in the series representation (17). A potentially better way to evaluate the kernel $\hat{K}(r)$ might be to use an $N$-point Gauss quadrature formula with weight function $t^{d-2}$ over the interval $[0,1]$. Denoting the nodes and weights by $t_{k}$ and $w_{k}$ respectively, the integral (16) can then be approximated as

$$
\hat{K}(r) \approx \sum_{k=1}^{N} w_{k}\left(\frac{e^{-j k_{0} r t_{k}}-1}{j k_{0} t_{k}}\right)
$$

In contradistinction with formula (17), where the delay aspects are completely blurred, it is seen that the delay information is still acutely present in formula (18).

\section{CONCLUSION}

Starting with the singular kernel present in the integrand, and making use of a powerful Gauss divergence theorem and a new Gauss bi-divergence approach, we have transformed the $2 d$-dimensional moment integral into a $(2 d-2)$-dimensional boundary integral. This allows in most pertinent cases to replace the original singular multiple integral by a simpler non-singular multiple integral, which can be evaluated by means of standard analytic or numerical quadrature techniques. Some pertinent special cases, such as the Coulomb and retarded Coulomb kernels, were shown to fit into the general scope of the proposed approach. 


\section{References}

[1] R. F. Harrington, Field Computation by Moment Methods. New York: Macmillan, 1968.

[2] S. M. Rao, D. R. Wilton, and A. W. Glisson, "Electromagnetic scattering by surfaces of arbitrary shape," IEEE Trans. Antennas Propagat., vol. AP-30, no. 3, pp. 409-418, May 1982.

[3] L. Knockaert, " A general Gauss theorem for evaluating singular integrals over polyhedral domains," Electromagnetics, vol. 11, pp. 269-280, 1991.

[4] A. H. Stroud, Approximate Calculation of Multiple Integrals. Englewood Cliffs, NJ: Prentice-Hall, 1971.

[5] F. Rellich, "Darstellung der Eigenwerte von $\Delta u+$ $\lambda u=0$ durch ein Randintegral.," Math. Z., vol. 46, pp. 635-636, 1940.

[6] L. Knockaert, F. Olyslager, and D. Vande Ginste, "On the evaluation of self-patch integrals in the method of moments," Microwave Opt. Technol. Lett., vol. 47, no. 1, pp. 22-26, Oct. 2005.

[7] G. Antonini, A. Orlandi, and A. E. Ruehli, "Analytical integration of quasi-static potential integrals on nonorthogonal coplanar quadrilaterals for the PEEC Method," IEEE Trans. Electromagnetic Compatibility, vol. 44, no. 2, pp. 399-403, May 2002. 